\title{
7. Interview mit Dr. Hans-Albert Treff, ICOM Deutschland-Präsident 1993-1998
}

\subsection{Interviewtext}

Dr. Albert Treff im Interview zu den Herausforderungen für ICOM Deutschland während seiner Amtszeit als Präsident (1993-1998), durchgeführt von Tanja Leiminger am Io. Februar 2020 in München:

Meine Amtszeit als Präsident von ICOM Deutschland begann im Jahr I993 und endete nach zwei Amtsperioden 1998 mit der Wahl meines Nachfolgers Prof. Dr. Hans-Martin Hinz.

Die Wiedervereinigung Deutschlands sowie die der beiden deutschen ICOM-Komitees lag gerade hinter uns. Spezielle allgemeine Herausforderungen waren damit jedoch nicht verbunden.

Damals schon bekannt, jedoch in der Museumswelt wenig thematisiert, war die Überprüfung von Restitutionsansprüchen.

Deutschland trat dem I970 gefassten UNESCO-Beschluss über »Convention on the Means of Prohibiting and Prevention the Illicit Import, Export and Transfer of Ownership of Cultural Property« erst im Jahr 2007 bei, sodass auch die mediale Aufmerksamkeit gegenüber diesem Thema zu dieser Zeit nicht groß war. Auf ICOM-Ebene fand die Diskussion aber statt, weshalb ich in meiner Rolle als ICOM-Präsident an einen Ausschuss im Bundestag schrieb, dass es empörend sei, sich dieser Resolution bisher noch nicht angeschlossen zu haben.

Ansonsten stand jedes Museum vor eigenen Problemen, denn trotz deren Boom in Deutschland litten auch zu meiner Amtszeit schon viele Häuser an finanziellen und personellen Engpässen.

Bei ICOM Deutschland waren wir zu meiner Amtszeit hauptsächlich mit organisatorischen Dingen, wie der Bereitstellung von Büroräumen und der Organisation des Komitees beschäftigt.

Ausgangspunkt dieser Umstrukturierungen waren handgeschriebene Karteikarten, die wir in Computersysteme einpflegten. Die Jahresberichte entwickelten wir von einem Brief am Ende des Jahres zu regelmäßig erscheinenden Mitteilungen weiter. Eine bessere Kommunikation mit den Mitgliedern wurde durch uns angeregt und mit den Jahren stetig verbessert, wodurch ste- 
tiges Mitgliederwachstum und mehr Zusammenarbeit unter den Kolleginnen und Kollegen entstand.

Trotzdem bestand immer die Gefahr sinkender finanzieller Zuwendungen.

Obwohl das Bundesverwaltungsamt stets gut gewillt war, stellten sich einige Vorgänge als mühsam dar. Selbst die Finanzierung einer 450 Mark-Bürohilfskraft war damals noch mit viel Aufwand verbunden.

Am Ende meiner Amtszeit wurden alle Aktionen mit anschließendem Bericht geprüft, sodass Prof. Hinz nach mir eine unbeanstandete, tadellose Buchhaltung übernehmen konnte.

Tanja Leiminger: Wie denken Sie, dass ICOM heute mit den Herausforderungen umgehen sollte?

Nicht viel anders, als der Vorstand es macht. Man kann die heutige Zeit mit der damaligen nicht mehr vergleichen.

Heutzutage ist die gesellschaftliche Diskussion viel komplizierter, zum Teil fast unverständlich, wie der Streit um die Museumsdefinition auf der ICOMJahrestagung 20I9 in München. Früher war Pragmatismus gefragt, während heute zu meinem Unmut theoretische Diskussionen überwiegen.

Ich muss aber ehrlich bekennen, dass ich mich zu Beginn meiner Pension bei ICOM zurückgezogen habe und ich nicht mehr über diese Themen informiert bin.

\section{Was konnten Sie konkret während Ihrer Amtszeit bei ICOM umsetzen?}

Zur Erleichterung der internen Arbeit im Vorstand bauten wir eine mit Computerwesen funktionierende Infrastruktur auf. Für unsere Mitglieder führten wir die dreijährliche Bodenseetagung in Lindau, die unter Prof. Auer ihre Anfänge fand, fort. Organisatorisch stellten diese beiden Punkte die Hauptaufgabe der deutschen ICOM-Zentrale dar.

Inhaltlich lag die Hauptlast auf den internationalen Komitees, die unabhängig von der Vorstandschaft Programme erstellten. Wir bemühten uns, deren Arbeit stets mit unseren sehr begrenzten finanziellen Mitteln zu unterstützen.

Schon unter meinem Vorgänger Prof. Auer bahnte sich eine Kooperation mit anderen ICOM-Komitees an, da zu dessen Amtszeit die Inaktivität des deutschen Nationalkomitees von der ICOM-Zentrale in Paris kritisiert wurde. Im Jahr I993 ergriffen wir schließlich im Vorstand die Initiative und bemühten uns, zusammen mit ICOM Österreich das skandinavische Beispiel der Nordeuropäischen Vereinigung auf das zentrale Europa zu übertragen, weshalb wir eine Kooperation mit östlich benachbarten Ländern anstrebten. Aufgrund meiner guten Beziehungen zu dem damaligen Präsidenten des Schwei- 
zer Nationalkomitees erfragte ich auch dort eine Zusammenarbeit, die dieser vermutlich aufgrund fehlender Bezüge zu den betreffenden Ländern ablehnte.

So fand die Kooperation schließlich zwischen ICOM Deutschland, Österreich und den Ländern, die sich aus westlicher Sicht hinter dem eisernen Vorhang befanden, also Polen, Tschechien, Slowakei, Ungarn, Slowenien und Kroatien, statt. Diese Vereinigung stellte den Beginn von CEICOM, Central European ICOM, dar.

In diesem Rahmen konnten wir allerdings nur marginale Sachen realisieren, wie etwa die Übernahme der Reisekosten einer leider nur geringen Zahl osteuropäischer Mitglieder zu deutschen ICOM Tagungen. Geplante Partnerschaftsmuseen innerhalb von CEICOM konnten wir aufgrund mangelnden Interesses der Beteiligten nicht umsetzen. Letztlich führten unsere Bestrebungen also zu keiner nennenswerten Weiterentwicklung.

Prof. Dr. Hans-Martin Hinz führte CEICOM in diesem Stil weiter und verzeichnete durch seine internationale Vernetzung einerseits und einer gefestigteren ICOM-Basis andererseits größere Erfolge. So bemühten sich schließlich weitere ICOM-Komitees, nämlich die aus Russland und Rumänien, um einen Beitritt. Als sich die wirtschaftlichen Bedingungen im europäischen Osten an die unseren annäherten, wurde diese Kooperation beendet.

Eine wesentlich sinnvollere Maßnahme war meines Erachtens die Finanzierung einer Zeitschriftenausgabe zusammen mit dem slowakischen ICOMNationalkomitee. Da die Mittel für den Druck das Bundesinnenministerium stellte, kamen wegen der geplanten Durchführung im Ausland vielfach Widerstände auf. Diese zu entkräften, stellte für uns im Vorstand einen unglaublichen Arbeitsaufwand dar. Schließlich konnten wir die Instanzen des Bundes durchlaufen und Autorenverträge vergeben, woraufhin die Zeitschrift in Bratislava mit deutsch-englischen Artikeln gedruckt und dort erfolgreich veröffentlicht wurde.

Solch eine Publikation ist trotz kleiner Auflage für eine breite Masse einsehbar und daher ergiebiger als vorherige Projekte, mit denen wir nur wenige Menschen erreichen konnten.

Eine Neuerung im Verband war, dass Reisekosten eines Mitgliedes aus den Fachkomitees zu internationalen Tagungen nur dann bezuschusst werden, wenn im Anschluss dessen ein Bericht verfasst wurde. Ein Verfahren, das ICOM Deutschland bis heute weiterführt.

Zudem gestalteten wir unsere Mitteilungen fortschrittlicher, obgleich die einfachen Blätter von damals kein Vergleich zu den heutigen Ausgaben sind. Im Endeffekt stellten wir natürlich nur einen kleinen Schritt in der Entwicklung des Verbandes dar, aber das ist Evolution und Optimierung. 


\section{Wieso sahen Sie durch CEICOM keine nennenswerte Weiterentwicklung?}

Der Hauptgrund waren begrenzte Mittel, weshalb ICOM Deutschland die Reisekosten von nur etwa acht Personen pro Jahr übernehmen konnte. Damit erzielt man wenige Ergebnisse. Die strenge Limitierung der Einladungen hatte auch zur Folge, dass beispielsweise der Präsident eines anderen Nationalkomitees unser Förderprogramm wiederholt ausschließlich für sich selbst nutzte.

Meiner Einschätzung nach entstanden Kooperationen eher auf individueller Ebene in den Fachkomitees. Ein mir überaus wichtiger Aspekt dieses Verbandes ist genau dieser Austausch unter Museumskolleginnen und -kollegen, der im beruflichen Alltag eine Erleichterung darstellen kann.

ICOM stellt ein Forum zur Kommunikation bereit, das durch neue Entwicklungen, wie etwa der auf der Jahrestagung in München von ICOM Netherlands vorgestellten Community, noch weiter ausgearbeitet wurde.

Sie sprachen die Kritik seitens der ICOM-Zentrale in Paris an, wie gestaltete sich die Zusammenarbeit im Allgemeinen?

Das Sekretariat war der Meinung, dass Deutschland als größtes Nationalkomitee aktiver sein sollte. Vorwürfe sind jedoch schnell ausgesprochen und entscheidend ist letztlich, was zwischen den Mitgliedern passiert und wie diese von unserem Verband profitieren können. Die Aktivitäten, die wir mit den Osteuropäischen Ländern anstrebten, begrüßte die Zentrale in Paris sehr und sprach Lob aus.

Die Zusammenarbeit im Allgemeinen war also damals und auch in heutiger Rückschau stets zufriedenstellend.

Wenn Sie auf Ihre Amtszeit zurückblicken, was gelang Ihnen Ihrer Meinung nach besonders gut?

Mit Stolz erfüllt mich die Wahl meines Nachfolgers, Prof. Dr. Hans-Martin Hinz, sowie die Berufung Dr. York Langensteins in den ICOM-Vorstand. Dies hatte zur Folge, dass Dr. Langenstein nach Prof. Hinz die ICOM-Präsidentschaft antrat.

Speziell Prof. Hinz brachte die Entwicklung von ICOM Deutschland weit voran und leistete durch seine exzellente Vernetzung wichtige Arbeit. Als Funktionär ist er damit bestens geeignet und konnte folglich auch mit internationalen Kooperationen wie CEICOM weitaus bessere Ergebnisse erzielen. Zudem ist er ein Organisationstalent und durchdenkt theoretische Angelegenheiten sehr konzentriert, um auch museologische Ziele zu verwirklichen. 


\section{Wie würden Sie die Ziele Ihrer Arbeit beschreiben?}

Prof. Auer schlug mich aufgrund unserer engen Zusammenarbeit während seiner letzten Amtsjahre für das Amt des Präsidenten von ICOM Deutschland vor. Mein Ziel war es, die von ihm geschaffene positive Grundstimmung in der Zusammenarbeit bei ICOM Deutschland beizubehalten. Ausufernde Diskussionen, wie sie zum Teil heute geführt werden, gab es zu dieser Zeit weder auf Mitglieder- noch auf Vorstandsebene.

Ich nahm mir vor, meine Amtszeit auf die gleiche lockere Art und Weise zu gestalten, wegen der sich Prof. Auer großer Beliebtheit erfreute. Zu Anfang der ersten Mitgliederversammlung unter meiner Leitung 1995 in Erfurt beispielsweise las ich die wunderbare Geschichte eines tschechischen Poeten vor, die dieser zu einem der Exponate meiner Ausstellung »Bärenstark. Natur- und Kulturgeschichte der Bären« verfasste.

So versetzte ich die ICOM-Mitglieder in gute Laune, was auch Streitereien untereinander verhinderte.

\section{Gibt es auch etwas, das Sie in Ihrer Rolle als Präsident von ICOM Deutschland eher hätten angehen sollen?}

Ich kann guten Gewissens auf meine Amtszeit zurückblicken, da ich stets im Rahmen meiner Möglichkeiten handelte, der letztlich jedoch festgelegt war. Einmal in drei Jahren fand die Bodenseekonferenz in Lindau statt, deren Vorbereitung und Kommunikation mit ICOM Österreich und Schweiz unglaublich zeit- und arbeitsintensiv waren. Ich legte stets Wert auf Lindau als Tagungsort und dem deutschen Nationalkomitee als Veranstalter. Eine Tradition, mit der zu meinem Bedauern nach meiner Amtszeit gebrochen wurde. Der Tagungsort und das austragende ICOM-Komitee wechselt nun im Dreijahrestakt zwischen ICOM Deutschland, Österreich und Schweiz.

Die wichtigste Aufgabe von ICOM ist ohnehin das Zusammenbringen der Museumskolleginnen und -kollegen. Ich selbst konnte als Mitglied wichtige nationale und internationale Kontakte knüpfen, die meine Arbeit im Museum erleichterten. Ohne das Forum zur Kommunikation, das ICOM bietet, kommen solche Beziehungen, die aus meiner Sicht der größte Gewinn sind, gar nicht erst zustande. 


\subsection{Kommentierung durch die Interviewerin}

Das Interview fand in der Privatwohnung von Dr. Hans-Albert Treff in München statt. Um eine ungestörte Gesprächssituation zu gewährleisten, bereitete dieser sein Wohnzimmer dementsprechend vor. Die Begrüßung war offen und freundlich, dennoch machte er bereits zu Beginn deutlich, dass er seit seiner Pensionierung die aktuellen Diskussionen die ICOM und das Museumsgeschehen betreffen nicht mehr aktiv verfolgt und diese der jüngeren Generation überlassen möchte. Diese Aussage bekräftigte er auch im Laufe des Interviews des Öfteren mit kurzen, nicht abgedruckten Kommentaren. Die Beantwortung der Fragen nahm einige Zeit in Anspruch und die Erzählstruktur war geprägt von späteren Ergänzungen und Einwürfen, die in der Überarbeitung des Interviews an der dementsprechenden Stelle ergänzt wurden, was aber keinesfalls negativ zu werten ist. An diesen spontanen Antworten wurden auch im Nachhinein keine sinnverändernden Eingriffe vorgenommen und nichts hinzugefügt, was sich nicht aus dem Gespräch ergab. Das vorliegende Interview wurde lediglich redaktionell bearbeitet und in die von ICOM Deutschland e.V. gewünschte Form gebracht. Gleichzeitig vermittelte Treff nicht den Eindruck, nicht unterstützen zu wollen, suchte bereitwillig Dokumente zur Veranschaulichung seiner Aussagen zusammen und lieferte im Gespräch nicht eindeutig geklärte Sachverhalte nach. Zögern und langes Überlegen bereitete vor allem die Einstiegsfrage nach Herausforderungen für die Museen im In- und Ausland sowie gesellschaftspolitische Aspekte, die ihm in seiner Amtszeit als Präsident von ICOM begegneten. Er legte dar, dass es zu dieser Zeit außer den allgemeinen Schwierigkeiten, die jedes Museum hinsichtlich Personal und Finanzierung hatte, keine nennenswerten gemeinsamen Probleme gab. Vielmehr kam dadurch und auch im weiteren Verlauf des Interviews der Eindruck zustande, dass unter Dr. Treff elementare Grundsteine für die interne Weiterentwicklung des Verbandes, wie etwa die Vorarbeit an der Bereitstellung der Büroräume, gelegt wurden. Besonders betonte er die geringeren finanziellen Zuwendungen und Handlungsspielräume, die ihm zur Verfügung standen. Durch seine Ausführungen vermittelte er den Eindruck, als sei er eine Person, die den Pragmatismus höher schätzt als theoretische Diskussionen um den Verband. Dass er zudem die notwendigen Aufgaben nach bestem Wissen und Gewissen und natürlich auch erfolgreich erledigte, die Präsidentschaft bei ICOM aber doch nur als Ehrenamt sah und sich aufgrund dessen darüber hinaus nicht aus Eigeninitiative um mehr Darstellung des Verbandes bemühte. Vielmehr war ihm an der Wahrung einer positiven Grundstimmung gelegen, die das Netzwerken unter den Mitgliedern unterstützen sollte. Sehr bescheiden, fast etwas zu Unrecht machte er zudem 
immer wieder deutlich, dass ihn die Wahl seiner Nachfolger am meisten mit Stolz erfülle. Das Interview verlief insgesamt sehr positiv. Die Gesprächssituation war wegen der Spontaneität der Antworten stets locker. Obwohl der Einstieg, wie bereits erwähnt, etwas holprig war, gelang nach ein paar Rückfragen ein aufrichtiges, fließendes Gespräch. Auch die Zusammenarbeit im Nachgang war unkompliziert und ging zügig vonstatten. 
